\title{
The role of wearables in spinal posture analysis: a systematic review
}

Lauren Simpson ${ }^{1,2}$, Monish M. Maharaj ${ }^{1,2,3,4^{*}}$ (I) and Ralph J Mobbs M $^{1,2,3}$

\begin{abstract}
Background: Wearables consist of numerous technologies that are worn on the body and measure parameters such as step count, distance travelled, heart rate and sleep quantity. Recently, various wearable systems have been designed capable of detecting spinal posture and providing live biofeedback when poor posture is sustained. It is hypothesised that long-term use of these wearables may improve spinal posture.
\end{abstract}

Research questions: To (1) examine the capabilities of current devices assessing spine posture, (2) to identify studies implementing such devices in the clinical setting and (3) comment on the clinical practicality of integration of such devices into routine care where appropriate.

Methods: A comprehensive systematic review was conducted in adherence to the Preferred Reporting Items for Systematic Reviews and Meta-Analyses Guidelines (PRISMA) across the following databases: PubMed; MEDLINE; EMBASE; Cochrane; and Scopus. Articles related to wearables systems able to measure spinal posture were selected amongst all published studies dated from 1980 onwards. Extracted data was collected as per a predetermined checklist including device types, study objectives, findings and limitations.

Results: A total of 37 articles were extensively reviewed and analysed in the final review. The proposed wearables most commonly used Inertial Measurement Units (IMUs) as the underlying technology. Wearables measuring spinal posture have been proposed to be used in the following settings: post-operative rehabilitation; treatment of musculoskeletal disorders; diagnosis of pathological spinal posture; monitoring of progression of Parkinson's Disease; detection of falls; workplace occupational health and safety; comparison of interventions.

Conclusions: This is the first and only study to specifically review wearable devices that monitor spinal posture. Our findings suggest that currently available devices are capable of assessing spinal posture with good accuracy in the clinical setting. However, further validation regarding the long-term use of these technologies and improvements regarding practicality is required for commercialisation.

Keywords: Wearable technology, Postural assessment, Patient outcomes, Spine posture

\section{Introduction}

Wearables are defined as electronic technologies or computers that can be worn on the body. Since the beginning of the twenty-first century such devices have reduced in size and costs while improving in observation capabilities related to multiple health-related parameters, triggering a shift towards commercialisation and incorporation into everyday activities [1]. In 2016, there were 61-million wearables monitoring physical activity online,

\footnotetext{
* Correspondence: monish.maharaj@gmail.com

${ }^{1}$ NeuroSpine Surgery Research Group (NSURG), Sydney, Australia

${ }^{2}$ Faculty of Medicine, University of New South Wales, Sydney, Australia

Full list of author information is available at the end of the article
}

forecasted to reach 187 million by 2020 [2]. The increased uptake of these devices has contributed to the self-monitoring movement of health. Most current wearable systems are accompanied by smartphone applications that permit users to engage and promote self-awareness of their behaviours as a means of motivating improvement and taking personal responsibility for their health [3]. Outside of physical activity, newer systems include observation of heart rate, blood pressure, posture and sleep [4]. This present review aims to focus on wearables analysing spinal posture and its health-related implications.

There is a need for real-time postural monitoring and correction as sustained poor spinal posture is associated

(c) The Author(s). 2019 Open Access This article is distributed under the terms of the Creative Commons Attribution 4.0 International License (http://creativecommons.org/licenses/by/4.0/), which permits unrestricted use, distribution, and reproduction in any medium, provided you give appropriate credit to the original author(s) and the source, provide a link to the Creative Commons license, and indicate if changes were made. The Creative Commons Public Domain Dedication waiver (http://creativecommons.org/publicdomain/zero/1.0/) applies to the data made available in this article, unless otherwise stated. 
with the development and worsening of many musculoskeletal disorders $[4,5]$. Poor spinal posture, as defined by Hansraj et al. [6], relates to the relative position whereby the head and upper trunk is in a forwardsflexed position. Neutral posture is considered when the head and upper trunk is at zero degrees to the rest of the spine, with subsequent increase in angle correlating with poorer posture and its associated complications. [6]. These complications are hypothesized to included improper alignment of vertebrae, intervertebral disc damage/degeneration and nerve root impingement. Clinically, this may be linked to symptoms including neck and back pain, radiculopathy and sensorimotor deficit. In severe symptomatic case this may warrant surgical intervention [7]. Ultimately, poor posture can lead to a significant economic burden through increased healthcare costs and lost productivity within the workforce [4]. While it is difficult to estimate the total cost of poor posture alone, the predicted burden of back pain in the United States alone as estimated by Shekelle et al. [8] is an annual net cost of $\$ 60$ billion.

Wearables monitoring posture have the potential to prevent the aforementioned consequences through real-time biofeedback encouraging the correction of sustained poor posture. It is hypothesised that with longterm use these systems may instil correct postural habits and yield a decrease in the incidence of posture-related musculoskeletal disorders. In a systematic review by Wang et al. [9] focused on sensor technology feedback systems of the upper limb ( $n=42$ studies) overall validation studies for multiple systems have been favourable, although clinical integration of these systems has not yet materialised on a large scale. In a separate review by McCallugh et al. [10] in the ageing and hospitalised population, a subset analyses on spinal posture across 6 devices had suggested high accuracy readings, although the use of combined feedback systems to target change and rehabilitation was generally lacking and remains an ongoing focus. The study was limited to devices detecting changes in sitting and standing posture, with further analyses outside the scope of the published review.

Conventionally, spinal posture is assessed in a clinical setting during routine patient examinations, physiotherapy sessions or formalised laboratory based evaluation. Such traditional methods have been criticised as costly and impractical with a key inability to measure day-to-day posture and provide timely feedback. The gold standard for analysing spinal posture remains radiographical assessment, however the associated cost and irradiation limits frequent repeated use [5]. Other lab-based modalities include: goniometers, photogrammetric systems and optoelectric systems may be utilised but have failed to integrate into routine medical practice.
This is the first study to review the use of wearables in spinal posture monitoring with aims to (1) examine the capabilities of current devices, (2) identify studies implementing such devices in a clinical setting and (3) comment on the clinical practicality of integration of such devices into routine clinical care. Our findings aim to synthesise the volume of data published across multiple scientific domains (engineering, computer science, rehabilitation medicine etc) and streamline a method of customised device design, in order to optimise the clinical use of postural sensors and ultimately use the data they can provide to facilitate clinical decision making such as the need for surgical intervention.

\section{Methods}

\section{Literature search}

The Preferred Reporting Items for Systematic Reviews and Meta-Analyses Guidelines (PRISMA) were adhered to for this systematic review across the following five databases: PubMed; MEDLINE; EMBASE; Cochrane; and Scopus [11]. The final choice of key search terms was derived from pre-established headings on the OVID Medline database after a generic screening using a list of relevant key terms. Key search terms included: 'Monitoring, Ambulatory' OR 'Wearable Electronic Devices' OR 'Wearables' AND 'Posture'. Relevant MeSH (Medical Subject Heading) terms, spelling variations and synonyms were included and modified as appropriate for each database. Studies addressing both wearable systems and posture were selected. The PRISMA flow chart is illustrated in figure.

\section{Study selection}

Inclusion and exclusion criteria are documented below. Duplicate studies were removed, with journal papers chosen over conference papers in the setting of data duplication. In cases where multiple studies included the same dataset, the most recent update was included. A primary screening was conducted by an independent reviewer (LS) who read the full texts of selected articles based on the inclusion and exclusion criteria; (4) A secondary screening was conducted by an independent reviewer (MM) who read the full texts of selected articles based on the inclusion and exclusion criteria. In the setting of discrepancy in study inclusion the senior author (RM) authorised the final decision.

Inclusion criteria:

1. Articles involving wearable technology/ies

2. The wearable technology/ies are able to monitor posture in the sagittal and/or coronal planes

3. The wearable technology/ies are able to monitor posture of the spine

4. Articles written in English

5. Time of publication between 1980- April 2018. 
Exclusion criteria:

1. Wearable technology/ies only capable of identifying activity or discriminating between body position (e.g. sitting, standing lying)

2. Wearable technology/ies only capable of monitoring posture of body parts other than the spine

3. Wearable technology/ies classed as robotic or exoskeletons

4. Systematic reviews

5. Books

\section{Data collection}

Following the selection of articles, data was collated by two researchers (LS and MM). Data was collected as per a predetermined checklist including: type of wearable technology/ies; sensor location/s; presence of feedback system; aims of the study; and outcomes of the study. All included articles were appraised for bias using the Newcastle-Ottawa Scale of Quality Assessment.

\section{Results}

From the 1427 non-duplicate articles found using the search strategy, 37 articles were selected for inclusion (Fig. 1). Summarised findings from selected articles are included in Table 1.

There were over 30 devices identified, with nineteen studies detailing real time feedback systems $(3,6,13$, $18-22,24-26,29,31,34,36,38-40,42)$. Among the included studies, ten studies had explicit aims of technological validation while most remaining studies others were limited as a proposal. Only seven studies employed the use of one sensor or single-platform with multiple sensors attached $(3,13,20,21,35,36,40)$, with the vast majority requiring multiple sensors worn at a single time. Parameters measures ranged from aspects of balance, spinal positioning, motion analyses and incidence of pre-defined postural positions. Two studies were explicitly focused on the analysis of spinal motion during chair-sitting exclusively $(6,38)$. One study had an aim to identify the optimal sensor positioning using a spine-based system as opposed to clinical validation (16). No studies included any meaningful cost analyses. When provided, the accuracy rates of devices were high with error ranges within 5 degrees in the majority of capturing moments ( $>85 \%$ of the time). Overall accuracy reporting is detailed later in the discussion.

Tables 2 and 3 detail the instruments used across studies within the wearable inertial units and the clinical populations in which testing was performed across the studies respectively.

Due to limitations in cohort differences and these recordable device parameters between studies it was not possible to meta-analyse data as a means of evaluating any expected postural based measures.

\section{Discussion}

Wearable technology

In 1994, Tanaka et al. proposed the first wearable system able to measure spinal posture without an observer. The

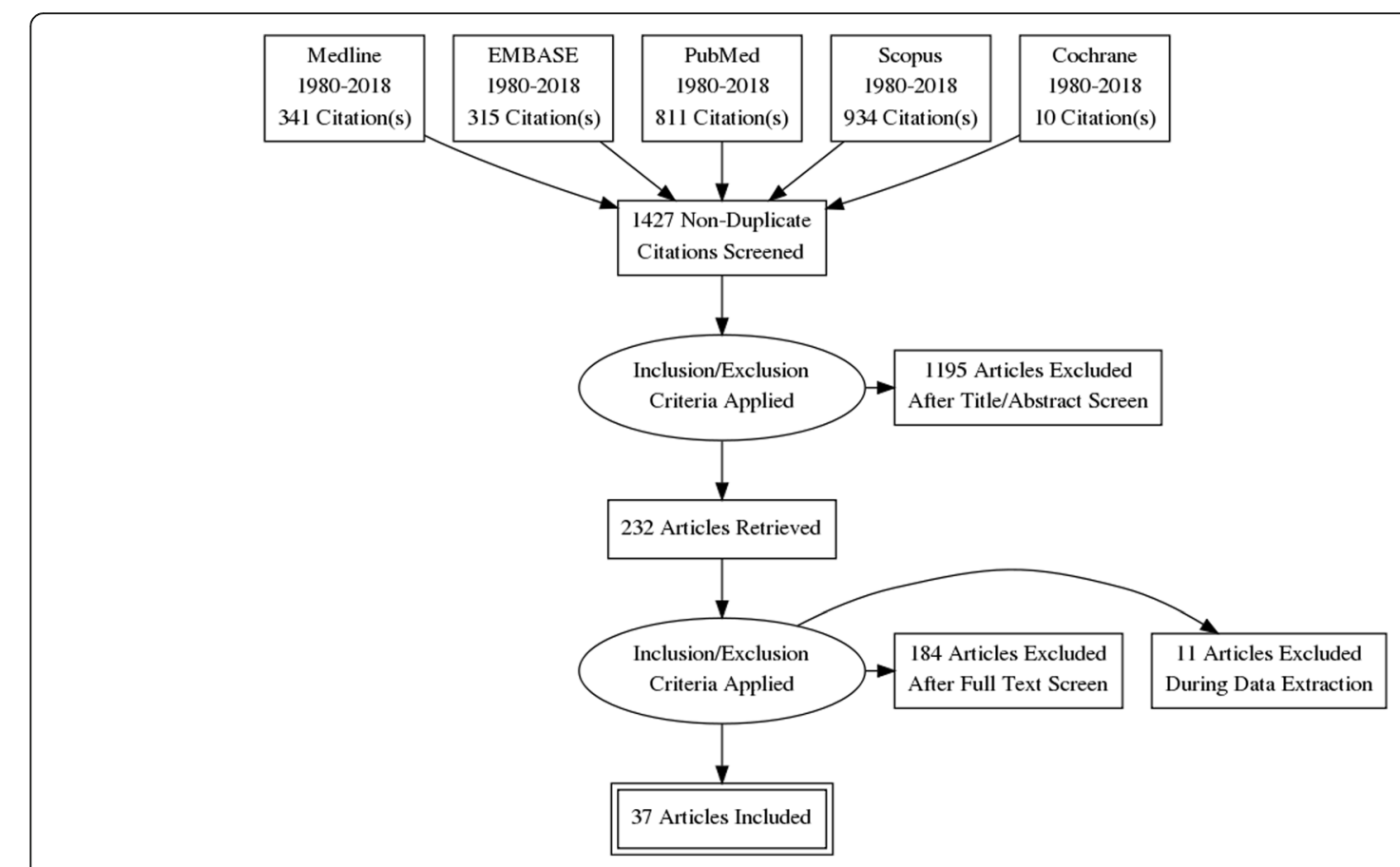

Fig. 1 PRISMA Flowchart outlining literature search and study selection 


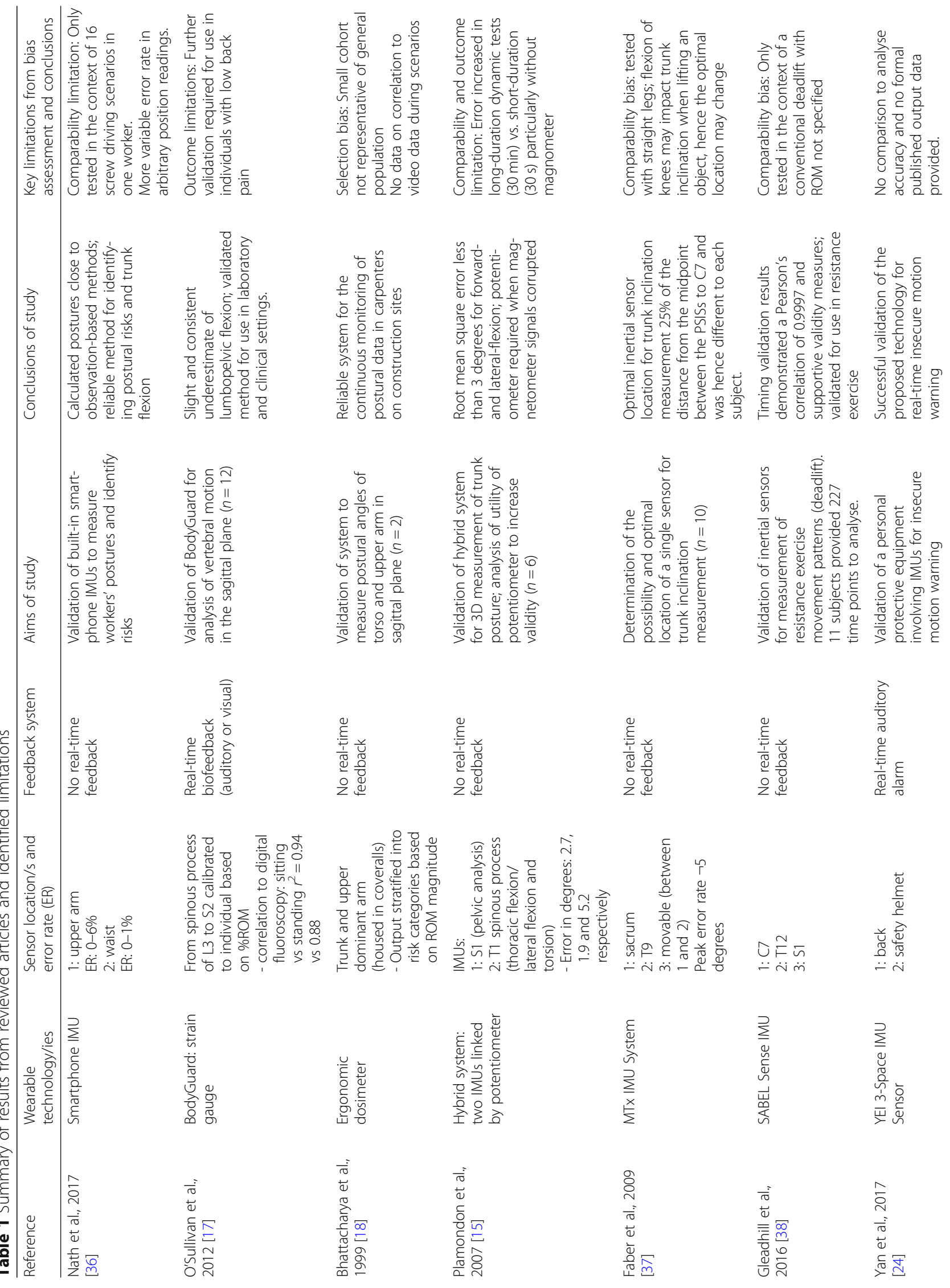




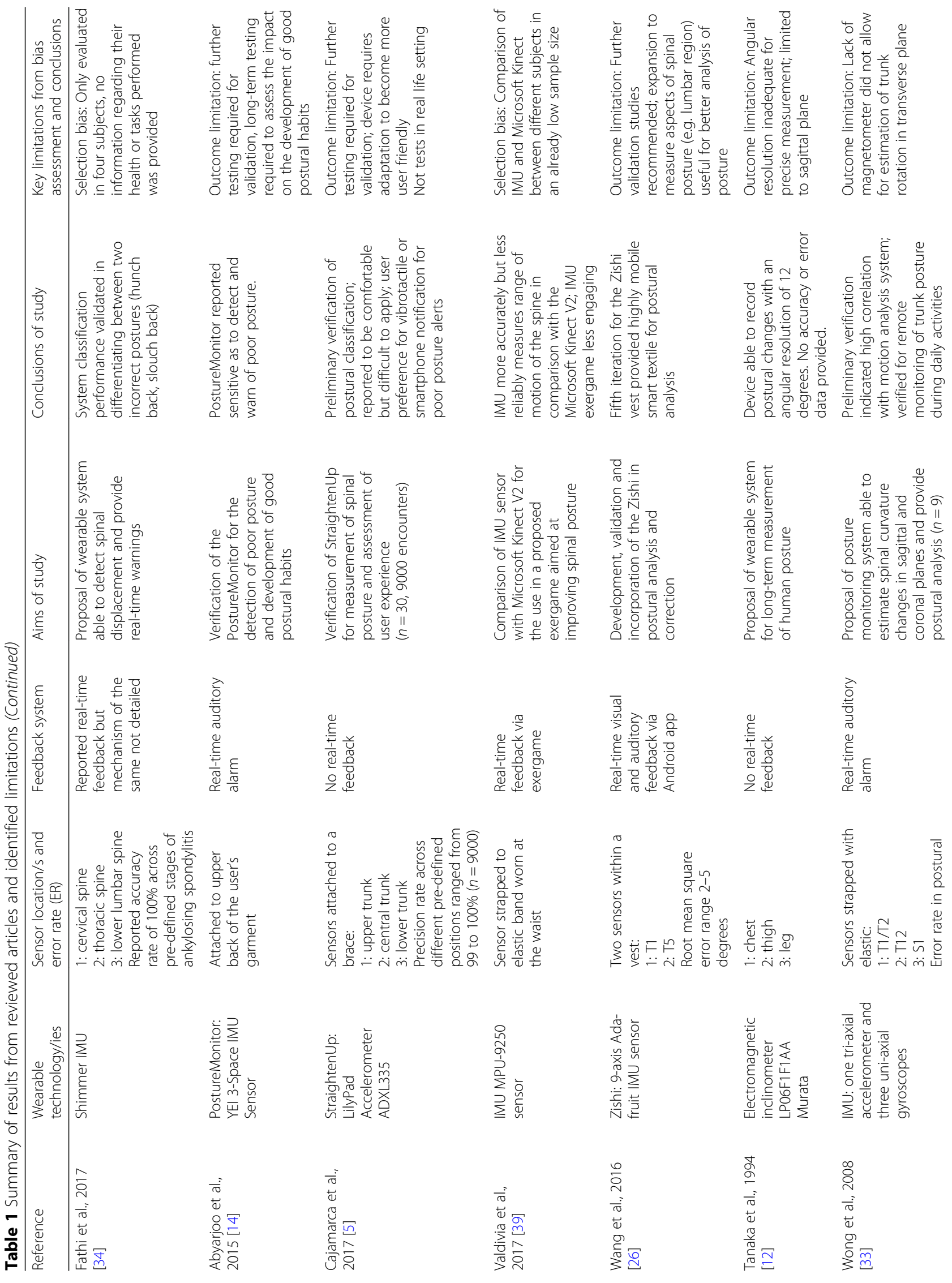




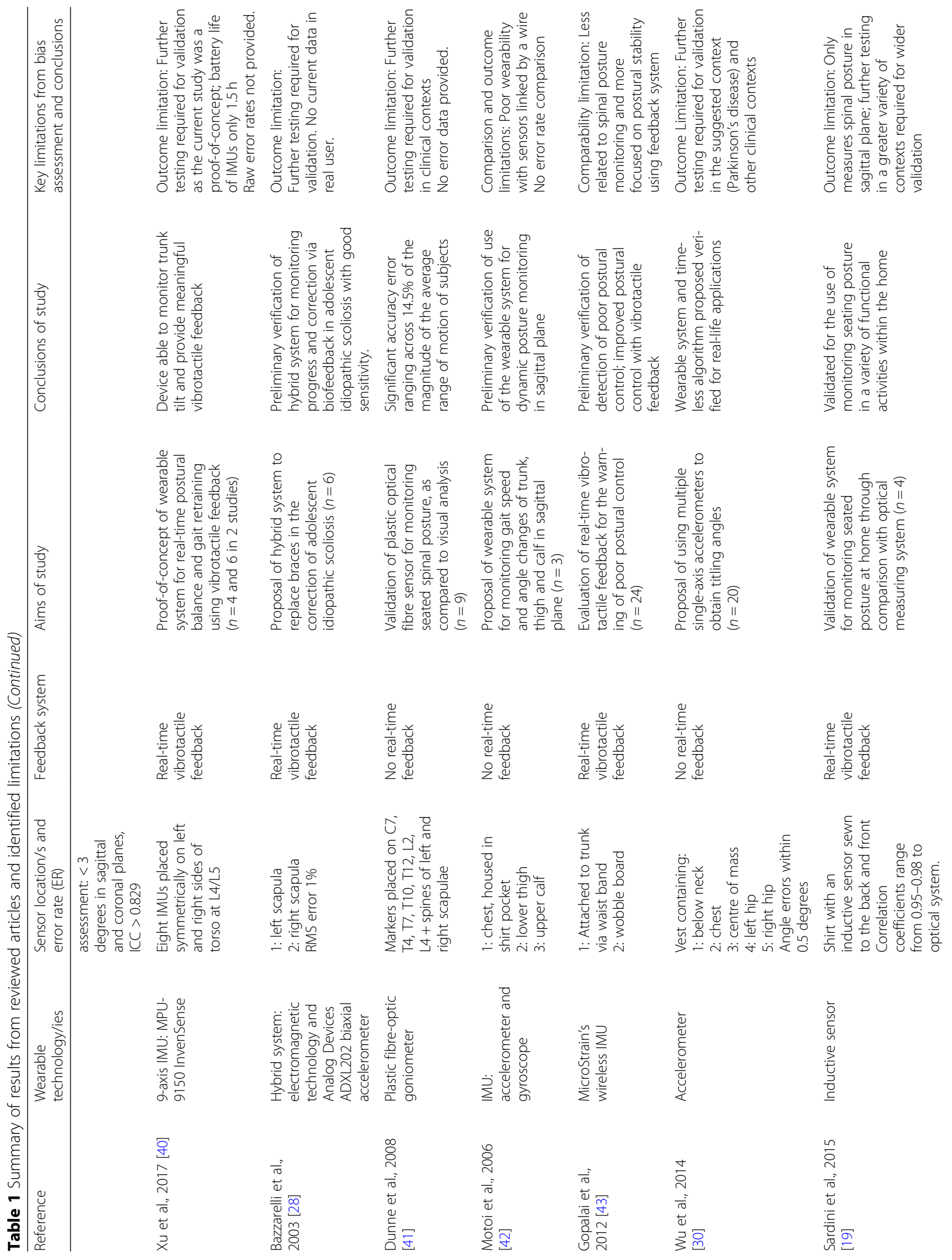




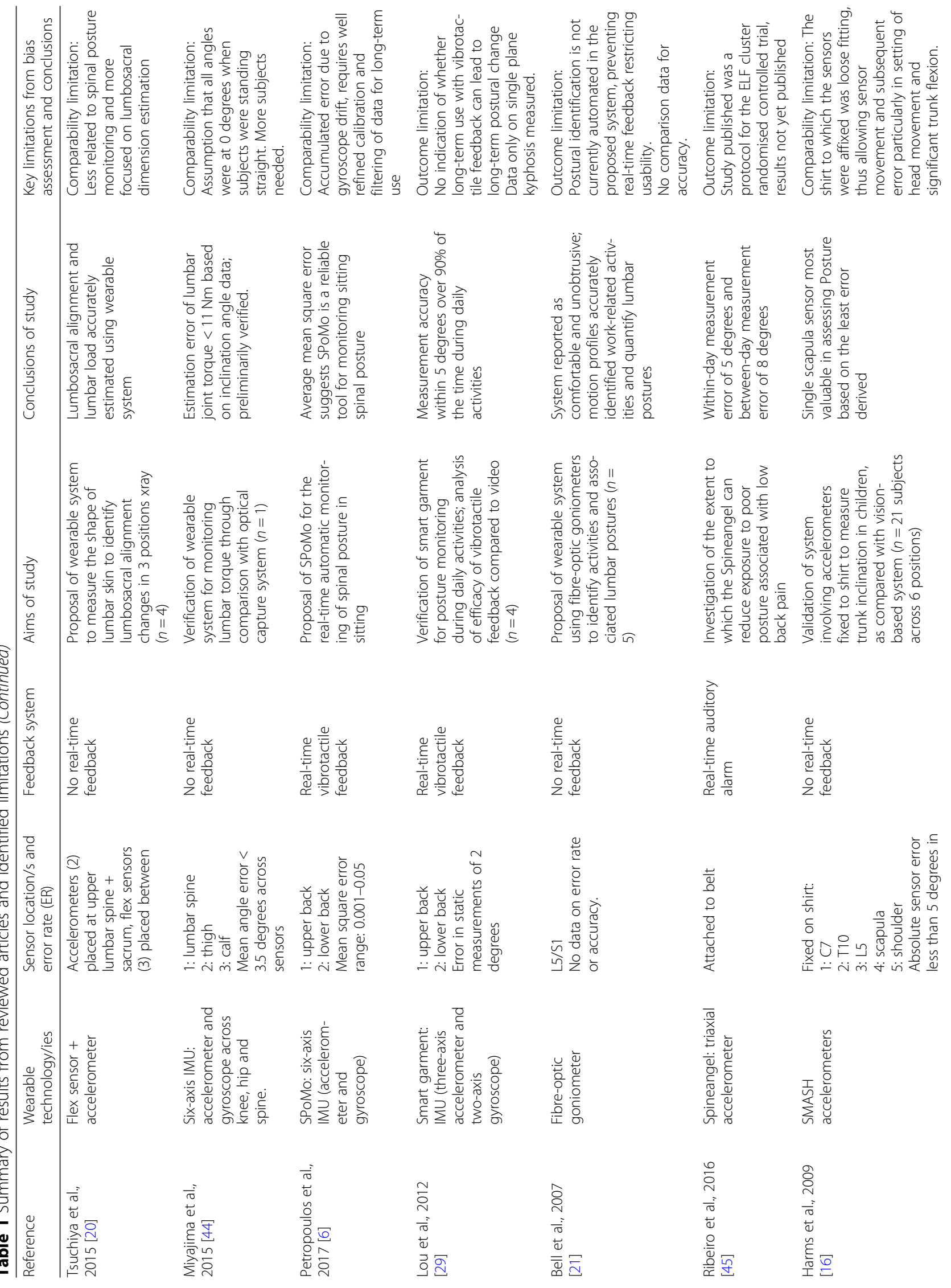




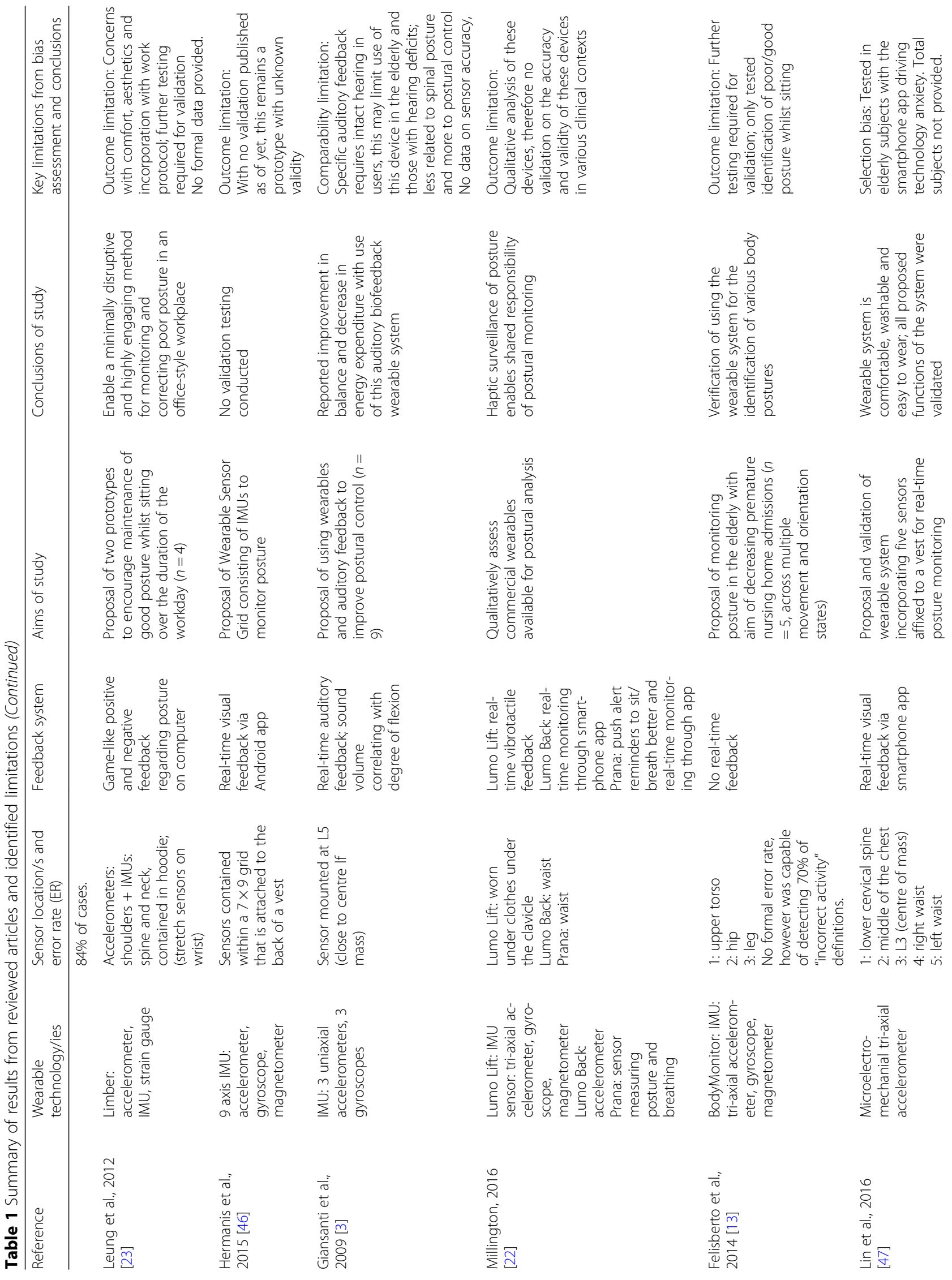




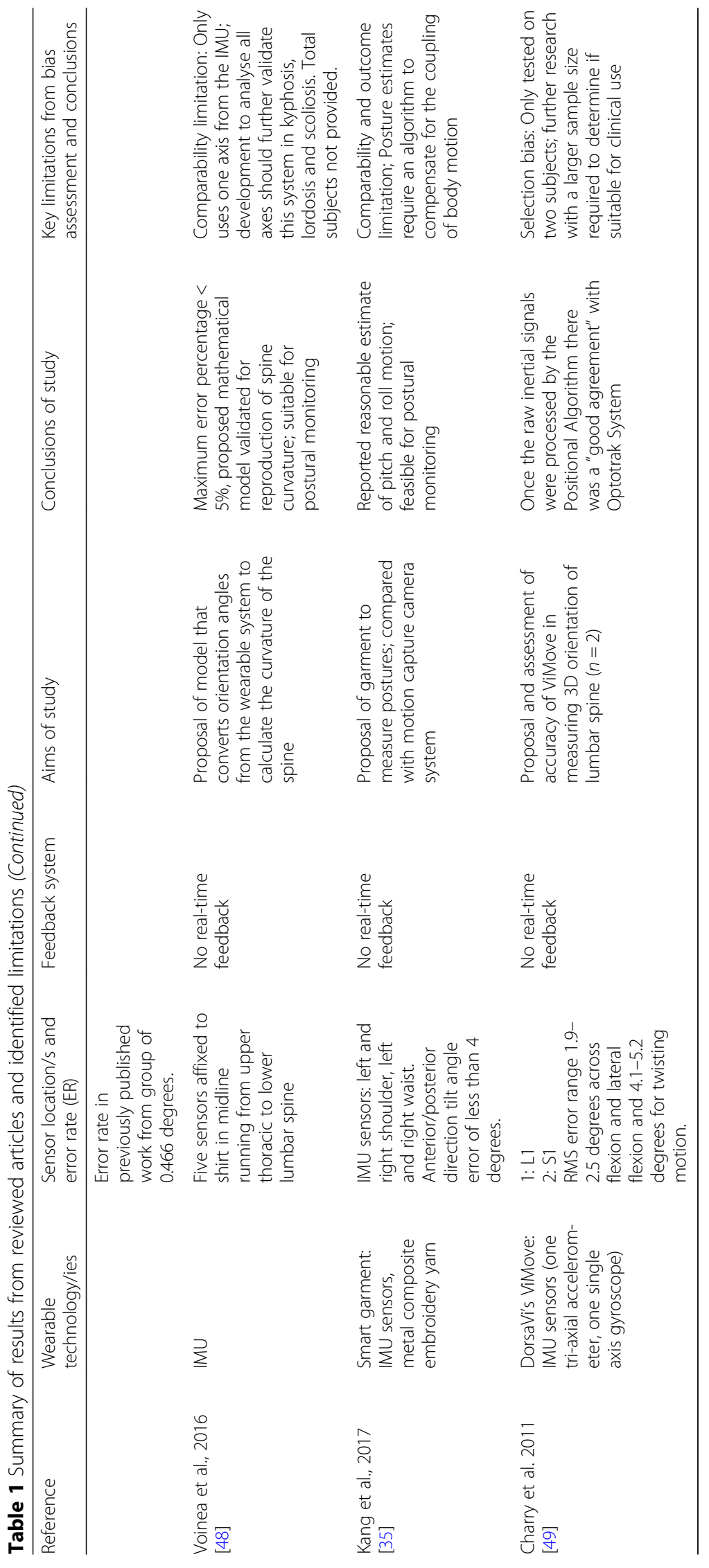


Table 2 Summary of IMUs

\begin{tabular}{|c|c|}
\hline \multicolumn{2}{|l|}{ Components } \\
\hline \multirow[t]{3}{*}{ Accelerometer } & Measure proper acceleration \\
\hline & - I.e. gravitational force (static) and sensor movement (dynamic) \\
\hline & - At least one 1D accelerometer \\
\hline \multirow[t]{2}{*}{ Gyroscope } & Measure angular velocity \\
\hline & - At least one 1D gyroscope \\
\hline \multirow[t]{2}{*}{ Magnetometer } & Measure all magnetic fields \\
\hline & - Optional \\
\hline \multicolumn{2}{|c|}{ Degrees of Freedom (DoF) } \\
\hline \multirow[t]{3}{*}{$9 \mathrm{DoF}$} & 3D accelerometer, 3D gyroscope \& 3D magnetometer: \\
\hline & $\begin{array}{l}\text { - Most accurate type of IMU as able to measure proper acceleration, angular velocity and } \\
\text { magnetic fields in three axes }\end{array}$ \\
\hline & $\begin{array}{l}\text { - Used in: PostureMonitor [34]; Zishi [39]; Xu's wearable system [33]; Hermanis' Wearable } \\
\text { Sensor Grid [23]; LumoLift [46]; BodyMonitor [22] }\end{array}$ \\
\hline \multirow[t]{3}{*}{6 DoF } & 3D accelerometer \& 3D gyroscope: \\
\hline & $\begin{array}{l}\text { - Less accurate than } 9 \text { DoF IMUs as no magnetometer, therefore lower accuracy in } \\
\text { determining sensor orientation }\end{array}$ \\
\hline & $\begin{array}{l}\text { - Used in: Giansanti's wearable system [3]; SPoMo [6]; Wong's wearable system [12]; } \\
\text { Miyajima's wearable system [20] }\end{array}$ \\
\hline \multirow[t]{3}{*}{5 DoF } & 3D accelerometer \& 2D gyroscope: \\
\hline & - Less accurate than 6 DoF IMUs as gyroscope cannot measure in the third dimension \\
\hline & - Used in Lou's Smart Garment [44] \\
\hline \multirow[t]{3}{*}{$4 \mathrm{DoF}$} & 3D accelerometer \& 1D gyroscope: \\
\hline & - Less accurate than 5 DoF IMUs as gyroscope can only measure in one dimension \\
\hline & - Used in DorsaVi's ViMove [35] \\
\hline
\end{tabular}

system comprised of three electro-magnetic inclinometers measuring angle of inclination in the sagittal field. The inclinometers were strapped to the chest, thigh and calf. An analog-to-digital converter was incorporated to transpose measured angles into 4-bit digital signals allowing digital reconstruction of the trunk, thigh and calf inclination angles. The proposed system had an angular resolution of 12 degrees, suitable for categorisation of posture as "poor" or "good". While Tanaka et al. reported "good linearity observed over the wide range of measured angle" there was no formal assessment of the accuracy of the system [12].

Since the proposal of such devices various prototypes capable of measuring spinal posture have been proposed. A wide range of technologies underpin these systems with the most commonly used being Inertial Measurement Units (IMUs). IMUs are generally comprised one or more accelerometers and one or more gyroscopes, and may also include one or more magnetometers (Table 2). Forces measured by accelerometers may be static, e.g. gravitational force, or dynamic, i.e. caused by moving or vibrating the accelerometer. The use of gyroscope permits precise measurement of angular velocity that is not influence by outer external forces detected
Table 3 Summary of posture wearable applicability

\begin{tabular}{|c|c|}
\hline \multicolumn{2}{|l|}{ Application } \\
\hline Post-operative rehabilitation [25] & Falls $[3,13,22]$ \\
\hline $\begin{array}{l}\text { - Reduced face-to-face hours } \\
\text { with rehabilitation provider }\end{array}$ & $\begin{array}{l}\text { - Detection of elderly falls within } \\
\text { the home }\end{array}$ \\
\hline - Tele-rehabilitation & $\begin{array}{l}\text { - Improvement of postural stability } \\
\text { to decrease the prevalence of } \\
\text { falls }\end{array}$ \\
\hline Treatment of MSK disorders & Workplace use \\
\hline $\begin{array}{l}\text { - Adolescent idiopathic } \\
\text { scoliosis [40] }\end{array}$ & - Office workers $[6,16]$ \\
\hline - Postural kyphosis [44] & - Construction workers [11.13] \\
\hline Diagnosis & Comparison of treatments \\
\hline $\begin{array}{l}\text { - Pathological spine postures } \\
\text { [24] }\end{array}$ & - E.g. spine operation types \\
\hline $\begin{array}{l}\text { - Assist in the clinical diagnosis } \\
\text { and rehabilitation of other } \\
\text { MSK disorders [9] }\end{array}$ & $\begin{array}{l}\text { - Through pre-operative and } \\
\text { post-operative monitoring [9] }\end{array}$ \\
\hline \multicolumn{2}{|l|}{$\begin{array}{l}\text { Monitoring of disease } \\
\text { progression }\end{array}$} \\
\hline - Parkinson's Disease [43] & \\
\hline
\end{tabular}


via accelerometry. The absolute nature of gyroscopes, however, raises an issue of bias error due to drift. Unlike accelerometers, which use the gravity vector as a reference, gyroscopes do not have a reference and therefore are unable to reset to an initial state, thus leading to an accumulation of errors. This may be reduced through magnetometer integration as these calibrate IMUs with reference to the Earth's magnetic field. However, as magnetometers cannot discriminate between the Earth's and other magnetic fields they may be prone to interference from hard iron distortions [13]. As a result, IMUs general contain a combination of all three monitors in all three vehicle axis planes (i.e. Euler Angles): pitch (x-axis); roll (y-axis); and yaw (z-axis) [14].

Some wearables couple IMUs with other technologies to improve overall accuracy of derived data. Plamondon et al. [15], proposed a wearable that combined two IMUs linked by a potentiometer. The potentiometer was shown to greatly improve the validity of the system by assessing the relative longitudinal rotation between the two IMU. The same study reported that the inclusion of the potentiometer reduced all root mean square error to $<5$ degrees, vastly improving data reliability [15]. This magnitude of error ( $<5$ degrees) appears to be the accepted standard throughout the literature. Our findings (Table 1) reveal a very high accuracy rate across different systems with error rates $<2$ degrees and high agreement to control methods, usually in the form of video footage or fluoroscopy. It may be speculated that these high accuracy findings are affected by publication bias against less accurate devices.

Other wearables use only accelerometers without the addition of gyroscopes or magnetometers. Harms et al. [16] proposed a system utilising five accelerometers affixed to a shirt in various locations. Three sensors were placed along the spinous processes of C7, T10 and L5, thus allowing for the detailed assessment of forwardflexion of the entire spine. A fourth sensor was placed at the scapula and the fifth was placed at a more superior aspect of the shoulder, thus allowing for measurement of lateral flexion [16]. However, the system proposed by Harms et al. [16] employed a loose fitting shirt and increasing movement of sensors along the skin yielding large measurement errors in the T10 and L5-located sensors. In particular, measurement errors increased with increased angle of inclination. Overall, their study suggested $>84 \%$ to represent an acceptable magnitude of sensor orientation error (i.e. percentage of samples at a specific angle of inclination within 5 degrees of true inclination). The proposed system is therefore only suitable for measuring posture between 0 and 20 degrees for forwards flexion. [16]. Other technologies used in posture monitoring wearables include: strain gauges; flex sensors; fibre-optic goniometers; inductive sensors; ergonomic dosimeters [17-21]. These devices are briefly explored in Table 1, however at this stage are not validated for routine clinical use.

\section{Clinical applicability}

Wearables measuring spinal posture have many possible clinical applications in the prevention, monitoring and treatment of chronic disease (Table 3). Mass production of smartphones, tablets and laptops have led to their incorporation into everyday life [22]. In office workers, long hours spent sitting in front of computers inevitably leads to poor posture. Ergonomic strategies to maintain correct posture, e.g. standing desks, have somewhat helped to reduce occupational risks to posture, however the unconscious deviation from intended correct posture is inevitable [23]. Wearable systems with the ability to monitor posture and provide real-time feedback alerting of sustained poor posture enable workers to correct their posture and hence decrease total time in poor postural states [9]. Abyarjoo et al. [14] have proposed an IMU-based wearable system for office workers that was reported to be sensitive enough to warn the user of poor postural states. This study was only a proposal and preliminary verification of the prototype, with studies assessing its validation in the office worker setting ongoing. This occupational environment represents a key health domain where such sensors may yield reduction in degenerative and traumatic postural changes on the basis of wearable derived preventative strategies. Such workers, particularly in the construction industry, are regularly exposed to repetitive strained postures and heavy lifting, establishing a clear focus population for future research. Yan et al. (2017) have proposed a wearable system with accompanying smartphone application producing auditory alerts upon detection of poor posture in the head, neck or trunk for longer than the acceptable holding time [24]. Like the system proposed by Abyarjoo et al. [14], this tool is also pending clinical validation.

Wearables measuring posture have a great potential for use in physical rehabilitation. Low back pain and other musculoskeletal disorders often require significant long-term rehabilitation to strengthen muscles and counteract postural deviations. Monitoring of posture using wearables may help to assist physical therapists ensure patients are properly executing rehabilitation exercises [25]. Furthermore, wearables may allow for reduced supervision by health professionals and the introduction of tele-rehabilitation via video conferencing. By using a wearable system at home, a physical therapist may be able to instruct and remotely monitor a patient's posture in real-time. Tele-rehabilitation is likely to have the biggest use in those geographically far from medical services and those who struggle to leave their residence (e.g. the 
elderly) [26]. A systematic review of tele-rehabilitation by Kairy et al. [27] reported that patients and therapists perceived tele-rehabilitation as useful and convenient. It was also reported that tele-rehabilitation was less costly for the healthcare system, however a cost analysis was not provided.

Postural wearables have the potential to become the primary treatment modality for some musculoskeletal disorders. Bazzarelli et al. [28] proposed a wearable system for treatment of adolescent idiopathic scoliosis (AIS). Traditionally, AIS is treated using braces that exert passive force on the spine causing the wearer to actively pull away from induced pressure points. It is reported that the active muscle contraction induced is the most important therapeutic component. The system proposed enforced this active contraction through vibrotactile feedback that was shown to be effective in encouraging postural correction in those with AIS and claim a device error rate $<1 \%$ for measurements in the angular range of 0 and 70 degrees [28]. Lou et al. [29] have proposed a similar device utilising IMU sensors affixed to a garment for the treatment of postural kyphosis in adolescents. Like AIS, postural kyphosis is primarily managed with aggressive bracing aiming to strengthen back muscles and increase spinal flexibility. Their findings highlighted an excellent correlation (pearson co-efficient $>0.999 ; p<0.05$ ) in the comparison of sensor data to a standardised rotating wheel apparatus [29]. However although both systems have demonstrated adequate accuracy, data assessing long-term outcomes and longitudinal clinical use remains lacking. There are no current studies demonstrating the impact of such devices on correction of posture.

Other clinical applications for postural monitoring wearables include: detection of falls within the home; prevention of falls through improved postural control; assessment of Parkinson's Disease severity [13, 16, 30]. The objective nature of wearables allows for their use in the aforementioned applications without the concern for subjective bias [31, 32]. With significant validation of postural wearable systems it may be possible for their use in the diagnosis of pathological spinal posture and the comparison of treatment modalities through the assessment of pre- and post-treatment postures [33].

\section{Practicality}

While many of the proposed wearables promise the potential use in a wide variety of clinical applications, the biggest challenge remains in the lack of validation of these technologies. Most of the reviewed articles either solely proposed prototype designs or conducted preliminary verification of devices using very small samples over a short-term of time. In order to validate their use for long-term postural monitoring and improvement, larger and longer duration validation studies are required.

Compared to their physical activity monitoring counter parts, wearables measuring posture are significantly lagging behind in terms of commercialisation. While the aforementioned lack of validation studies is a major cause, the need for more than one sensor in determining spinal posture presents a significant challenge regarding practicality. Although some of the wearable systems proposed only used one sensor, Fathi et al. [34] reported an optimum number of three IMU sensors for accurate classification of posture. Their findings indicated that the addition of more than three sensors did not achieve a statistically significant improvement in the accuracy of postural analysis data [34]. Multiple sensors require connection via wires and attachment to the body, often by strapping they may be challenging for the user to remove and reattach [26]. The key to the commercialisation of these devices is finding the perfect middle ground between accuracy and wearability. Hence, it is understood that this is the rationale for the popular choice of three sensors as seen in many of the proposed devices.

One method to make multiple-sensor wearables more practical is the incorporation of sensors into smart textiles. Kang et al. [35], proposed a wearable garment that contained four IMUs incorporated within stretchable conductive yarn. The conductive yarn functioned to transmit signals from the sensors to the processor and also allow for battery power transmission. The wearable garment was reported to provide a reliable estimation of postural tilt of the torso over $1 \mathrm{~h}$. There was strong linearity demonstrated in pitch and roll directions, with both producing an $R^{2}>0.973$. There were, however, increased errors in measurements over $1 \mathrm{~h}$. It is suspected that this increase in estimation errors occurred due to the relative motion of IMU modules against the body [35]. Despite the stretchable nature of the fabric, future improvements to this system must address this issue of sensor movement.

Millington [22] reviews the three commercially available wearables able to monitor spinal posture: Lumo Back; Lumo Lift; Prana. The Lumo Back is an accelerometerbased sensor measuring $3.9 \mathrm{in}$. and is worn at the waist. It monitors lower back posture and encourages self-surveillance via a smartphone application but does not provide real-time warnings of sustained poor posture. The Lumo Lift is a smaller and more discreet device, measuring 1.74 in. and clasped under clothes just inferior to the clavicle. It measures upper back posture allows users to switch between posture alert mode, where vibrotactile feedback is provided in response to sustained poor posture, and coaching mode, where a vibration is delivered as soon as poor posture is assumed. The Lumo Lift also monitors 
step count, distance and energy expenditure. The Prana is a disc-shaped sensor measuring $1.25 \mathrm{in}$. that is worn at the waist. It measures breathing and posture and sends notifications reminding users to maintain neutral posture [22]. Millington's review of these three devices is only a qualitative analysis and lacks reference to significant quantitative validation. A comprehensive review of the literature also failed to find published validation trials for these devices. Hence, further research assessing their impact long-term is required.

As devices continue to improve in accuracy and achieve validation we believe the next step towards integration of such wearables would be to establish a link between data output and diagnostic predictability while optimising costs of integration. The implication a diagnostic algorithm in this regard would facilitate healthcare delivery without the need for regular consultation and the potential to remotely highlight populations requiring urgent intervention. This remains a key focus for our own research institution at this stage.

\section{Conclusion}

This is the only study to specifically review wearable devices that monitor spinal posture. This review reveals that spinal posture can be measured through the use of various technologies but that there is limited data regarding the validation of the same. More research into the accuracy and long-term outcomes of these devices is required for a greater understanding of their clinical applicability. Furthermore, improvements regarding practicality are required before commercialisation and mass uptake can be considered.

\section{Abbreviations}

AIS: Adolescent idiopathic scoliosis; DoF: Degrees of Freedom; IMUs: Inertial Measurement Units; PRISMA: Preferred Reporting Items for Systematic Reviews and Meta-Analyses Guidelines

\section{Acknowledgements}

There are no further acknowledgements.

\section{Funding}

There was no funding retrieved for the conduct of this study.

\section{Availability of data and materials}

All data generated or analysed during this study are included in this published article and its supplementary files (table file and figure file),

\section{Authors' contributions \\ MM and RJM were involved in the concept and design of the study. LS and MM conducted the literature review process as described in the methods section and final included studies were reviewed by all authors. LS was involved with data preparation and manuscript construction. MM and RM were involved in editing and reviewing the final manuscript. MM and RM supervised the study. All authors read and approved the final manuscript.}

\section{Ethics approval and consent to participate}

Not applicable

\section{Consent for publication}

Not applicable

\section{Competing interests}

The authors declare that they have no competing interests.

\section{Publisher's Note}

Springer Nature remains neutral with regard to jurisdictional claims in published maps and institutional affiliations.

\section{Author details}

${ }^{1}$ NeuroSpine Surgery Research Group (NSURG), Sydney, Australia. ${ }^{2}$ Faculty of Medicine, University of New South Wales, Sydney, Australia. ${ }^{3}$ Department of Neurosurgery, Prince of Wales Hospital, Sydney, Australia. ${ }^{4}$ Prince of Wales Hospital, Randwick, NSW, Australia.

Received: 20 August 2018 Accepted: 22 January 2019

Published online: 08 February 2019

\section{References}

1. Wei J. How wearables intersect with the cloud and the internet of things: considerations for the developers of wearables. IEEE Consumer Electronics Magazine. 2014:53-6.

2. CSS Insight. Market forecast wearables. 2016. Available from: https://www. ccsinsight.com/our-services/1711. Accessed May 2017.

3. Giansanti D, Dozza M, Chiari L, Maccioni G, Cappello A. Energetic assessment of trunk postural modifications induced by a wearable audiobiofeedback system. Med Eng Phys. 2009;31:48-54.

4. Piwek $L$, Ellis $D$, Andrews $S$, Joinson $A$. The rise of consumer health wearables: promises and barriers. PLoS Med. 2016;13(2):1-9.

5. Cajamarca G, Rodriguez I, Herskovic V, Campos M. Ubiquitous Computing and Ambient Intelligence. Santiago: Springer International Publishing; 2017. Available from: http://link.springer.com/10.1007/978-3-319-67585-5

6. Hansraj K. Assessment of stresses in the cervical spine caused by posture and position of the head. Surg Technol Int. 2014;25:277-9.

7. Petropoulos A, Sikeridis D, Antonakopoulos T. SPoMo: IMU-based real-time sitting posture monitoring. Patras; 2017

8. Shekelle P, Coulter I, Hurwitz E, Genovese B, Adams A, Mior S, et al. Congruence between decisions to initiate chiropractic spinal manipulation for low Back pain and appropriateness criteria in North America. Ann Intern Med. 1998;129(1):9-17.

9. Wang Q, Markopoulos P, Yu B, Chen W, Timmermans A. Interactive wearable systems for upper body rehabilitation: a systematic review. J Neuroeng Rehabil. 2017:14(20):1-21.

10. McCullagh R, Brady NM, Dillion C, Horgan NF, Timmons S. A review of the accuracy and utility of motion sensors to measure physical activity of frail, older hospitalized patients. J Aging Phys Act. 2016;24(3):465-75.

11. Moher D, Liberati A, Tetzlaff J, Altman D, Altman D, Antes G, et al. Preferred reporting items for systematic reviews and meta-analyses: the PRISMA statement. PLoS Med. 2009;6(7):1-6.

12. Tanaka S, Yamakoshi K, Rolfe P. New portable instrument for long-term ambulatory monitoring of posture change using miniature electro-magnetic inclinometers. Med Biol Eng Comput. 1994;32:357-60.

13. Felisberto F, Fdez-Riverola D, Pereira A. A Ubiquitous and low-cost solution for movement monitoring and accident detection based on sensor fusion. Sensors (Basel). 2014;14:8961-83.

14. Abyarjoo F, O-Larnnithipong N, Tangnimitchok S, Ortega F, Barreto A. PostureMonitor: real-time IMU wearable technology to Foster poise and health. Miami; 2015.

15. Plamondon A, Delisle A, Larue C, Brouillette D, Mcfadden D, Desjardins $P$, et al. Evaluation of a hybrid system for three-dimensional measurement of trunk posture in motion. Appl Ergon. 2007:38:697-712

16. Harms H, Amft O, Troster G, Appert M, Muller R, Meyer-Heim A. Wearable therapist: sensing garments for supporting children improve posture. Zurich; 2009

17. O'Sullivan K, Verschueren S, Pans S, Smets D, Dekelver K, Dankaerts W. Validation of a novel spinal posture monitor : comparison with digital videofluoroscopy. Eur Spine J. 2012;21:2633-9.

18. Bhattacharya A, Warren J, Teuschler J, Dimov M, Medvedovic M, Lemasters $\mathrm{G}$. Development and evaluation of a microprocessor-based ergonomic dosimeter for evaluating carpentry tasks 1. Appl Ergon. 1999:30:543-53.

19. Sardini E, Serpelloni M, Pasqui V. Daylong sitting posture measurement with a new wearable system for at home body movement monitoring. Brescia; 2015 
20. Tsuchiya Y, Kusaka T, Tanaka T, Matsuo Y, Oda M, Sasaki T, et al. Calibration method for lumbosacral dimensions in wearable sensor system of lumbar alignment. Sapporo; 2015.

21. Bell J, Stigant M. Development of a fibre optic goniometer system to measure lumbar and hip movement to detect activities and their lumbar postures. J Med Eng Technol. 2007:31(5):361-6.

22. Millington B. "Quantify the invisible": notes toward a future of posture. Crit Public Health. 2016;26(4):405-17.

23. Leung K, Reilly D, Hartman K, Stein S, Westecott E. Limber: DIY wearables for reducing risk of office injury. Toronto; 2012.

24. Yan X, Li H, Li A, Zhang H. Automation in construction wearable IMU-based real-time motion warning system for construction workers' musculoskeletal disorders prevention. Autom Constr. 2017;74:2-11.

25. Rednic R, Gaura E, Kemp J, Brusey J. Fielded autonomous posture classification systems: design and realistic evaluation. In: 14th ACIS international conference on software engineering, Artificatial intelligence, Netweorking and parallel/distributed Computing; 2013. p. 635-40.

26. Wang Q, Timmermans A, Toeters M, Markopoulos P, Chen W. Zishi: a smart garment for posture monitoring. The Netherlands; 2016.

27. Kairy D, Lehoux P, Vincent C, Visintin M. A systematic review of clinical outcomes, clinical process, healthcare utilization and costs associated with telerehabilitation. Disabil Rehabil. 2009;31(6):427-47.

28. Bazzarelli M, Durdle NG, Lou E, Raso V. A wearable computer for physiotherapeutic scoliosis treatment. IEEE Trans Intr Measur. 2003;52(1):126-9.

29. Lou E, Lam G, Hill D, Wong M. Development of a smart garment to reduce kyphosis during daily living. Med Biol Eng Comput. 2012;50:1147-54.

30. Wu W, Lin W, Lee M. Forward-flexed posture detection for the early Parkinson's disease symptom. In: IEEE International Conference on Systems, Man and Cybernetics; 2014. p. 1181-5.

31. Rao P, Phan K, Maharaj M, Pelletier M, Walsh W, Mobbs R. Accelerometers for objective evaluation of physical activity following spine surgery. J Clin Neurosci. 2016;26:14-8.

32. Mobbs R. Wearables in spine surgery: Beginnings, research and real-world applications. Spinal News Int. 2017; Available from: https://spinalnewsinternational.com/wearables.

33. Wong W, Wong M. Trunk posture monitoring with inertial sensors. Eur Spine J. 2008;17:743-53.

34. Fathi A, Curran K. Detection of spine curvature using wireless sensors. J King Saud Univ Sci. 2017;29(4):553-60.

35. Kang S, Choi H, Park H, Choi B, Im H, Shin D, et al. The development of an IMU integrated clothes for postural monitoring using conductive yarn and interconnecting technology. Sensors (Basel). 2017;17(2560):1-10.

36. Nath N, Akhavian R, Behzadan A. Ergonomic analysis of construction worker's body postures using wearable mobile sensors. Appl Ergon. 2017;62: 107-17.

37. Faber G, Kingma I, Bruijn S, van Dieen J. Optimal inertial sensor location for ambulatory measurement of trunk inclination. J Biomech. 2009;42:2406-9.

38. Gleadhill S, Bruce J, James D. The development and validation of using inertial sensors to monitor postural change in resistance exercise. J Biomech. 2016;49(7):1259-63.

39. Valdivia S, Blanco R, Penuela L, Rojas D, Kapralos B. A spinal column Exergame for occupational health purposes. Bogota; 2017.

40. Xu J, Bao T, Lee U, Kinnaird C, Carender W, Huang Y, et al. Configurable, wearable sensing and vibrotactile feedback system for real-time postural balance and gait training: proof-of-concept. J Neuroeng Rehabil. 2017; 14(102):1-10.

41. Dunne L, Walsh P, Hermann S, Smyth B, Caulfield B. Wearable monitoring of seated spinal posture. IEEE Trans Biomed Circuits Syst. 2008;2(2):97-105.

42. Motoi K, Ikeda K, Kuwae Y, Yuji T, Higashi Y, Nogawa M, et al. Development of an ambulatory device for monitoring posture change and walking speed for use in rehabilitation. In: IEEE EMBS Annual International Conference; 2006. p. 5940-3.

43. Gopalai A, Senanayake S, Lim K. Intelligent Vibrotactile biofeedback system for real-time postural correction on perturbed surfaces. Sarawak Campus; 2012.

44. Miyajima S, Tanaka T, Imamura Y, Kusaka T. Lumbar joint torque estimation based on simplified motion Measurement using multiple inertial sensors. Sapporo; 2015.

45. Ribeiro D, Milosavljevic S, Abbott J. Effectiveness of a lumbopelvic monitor and feedback device to change postural behaviour: a protocol for the ELF cluster randomised controlled trial. BMJ Open. 2017;7(1):-10.
46. Hermanis A, Cacurs R, Nesenbergs K, Greitans M, Syundyukov E, Demonstration Abstract SL. Wearable sensor grid architecture for body posture and surface detection and rehabilitation. Riga; 2015.

47. Lin W, Chou W, Tsai T, Lin C, Lee M. Development of a wearable instrumented vest for posture monitoring and system usability verification based on the technology acceptance model. Sensors (Basel). 2016;16(2172):1-11.

48. Voinea G, Butnariu S, Measurement MG. Geometric modelling of human spine posture for medical rehabilitation purposes using a wearable monitoring system based on inertial sensors. Sensors (Basel). 2017;17(3):1-19.

49. Charry $E$, Umer M, Taylor S. Design and validation of an ambulatory inertial system for 3-D measurements of low back movements. In: Proceedings of the 2011 7th international conference on intelligent sensors, sensor networks and information processing; 2011. p. 58-63.
Ready to submit your research? Choose BMC and benefit from:

- fast, convenient online submission

- thorough peer review by experienced researchers in your field

- rapid publication on acceptance

- support for research data, including large and complex data types

- gold Open Access which fosters wider collaboration and increased citations

- maximum visibility for your research: over $100 \mathrm{M}$ website views per year

At BMC, research is always in progress.

Learn more biomedcentral.com/submissions 